\title{
A rare case of pedunculated bronchial hemangioma
}

\author{
M. Bellia1, A. Lo Casto1, F. Guddo2, M. Midiri1, S. Regio3, \\ R. Lagalla1, V. Bellia4
}

ABSTRACT. A rare case of pedunculated bronchial hemangioma. M. Bellia, A. Lo Casto, F. Guddo, M. Midiri, S. Regio, R. Lagalla, V. Bellia.

We describe a rare case of pedunculated endobronchial hemangioma observed in a 60 years old patient complaining of chronic productive cough and accessional dyspnea which had been progressively worsening over 20 years. The lesion was first noticed at fiberoptic bron- choscopy; then computed tomography scan was performed and integrated with tridimensional reconstruction techniques. Pathology showed the picture of a vascular neoplasm, compatible with capillary hemangioma. The lesion was submitted to laser-assisted endoscopic removal in order to relieve the obstruction, leading to remission of symptoms.

Monaldi Arch Chest Dis 2008; 69: 4, 189-191.

Keywords: Bronchial hemangioma, Computed tomography, Pulmonary neoplasms.

${ }^{I}$ Dipartimento di Biotecnologie Mediche e Medicina Legale, Sezione di Radiologia, Università di Palermo;

2 U.O. di Anatomia Patologica, A.O. "V. Cervello", Palermo;

3 U.O. di Chirurgia Toracica, A.O. "V. Cervello", Palermo;

4 Dipartimento di Medicina, Pneumologia, Fisiologia e Nutrizione Umana, Sezione di Pneumologia, Università di Palermo, Italy.

Correspondence Prof. Vincenzo Bellia, Dipartimento di Medicina, Pneumologia, Fisiologia e Nutrizione Umana, Università di Palermo, clo Ospedale "V.Cervello",Via Trabucco 180,90146 Palermo, Italy; e-mail: v.bellia@unipa.it

\section{Introduction}

Bronchial localisation of hemangiomas is exceedingly rare both in childhood and in adult age. However it may occasionally result in problems of differential diagnosis [1]. Clinical picture may be characterised by cough and hemophtysis [2,3]; in cases of larger tumours, developing as endobronchial pedunculated or vegetating masses, atelectasis and severe dysventilatory phenomena may occur: therefore hemangiomas must be included among possible causes of bronchial obstruction, particularly in children [4].

The lesion may be diagnosed by fiberoptic bronchoscopy (FBS) and biopsy integrated by computed tomography (CT) scan. Recently the introduction of tridimensional reconstruction modalities allows the performance of a virtual bronchoscopy: this new imaging technique might provide a better definition of morphology and topography of lesions particularly when it is not possible to examine the portion of bronchial tree distal to the lesion. In these cases virtual bronchoscopy might document the condition of those bronchi that cannot be directly visualised by traditional FBS.

In the present study we report a case of a 60 year old patient with an endobronchial pedunculated hemangioma detected by FBS and then fully evaluated by spiral CT integrated with tridimensional reconstruction and virtual bronchoscopy.

\section{Case Report}

A 60 year old, male, caucasian patient was admitted into hospital because of exacerbation of chronic obstructive pulmonary disease (COPD). As a smoker of 45 packs/year, he reported a history of chronic productive cough and of accessional dyspnea progressively worsening over the preceding 20 years, systemic hypertension since 10 years. A CT scan of thorax revealed an endobronchial lesion, which at FBS appeared as localised in the right main and intermediate bronchi (figure 1). The patient was immediately invited to refer to a thoracic surgery centre for the treatment of the lesion. After the admission a new FBS assessment confirmed the previous findings that the right main and intermediate bronchi were nearly completely obstructed by a reddish, smooth surface protruding mass which did not seem to infiltrate the surrounding wall.

For a better planning of the surgical approach, particularly aimed at defining the caudal extension and the area of insertion of the mass, a further volumetric CT scan was performed by a Picker International UltraZ (Cleveland, Ohio, USA) spiral equipment. The test was carried out before and after the i.v. administration of contrast medium. After the evaluation of the axial transverse images and after selection of the portion of interest, the test continued with a second acquisition. Images were then transferred into Digital Images Communica- 


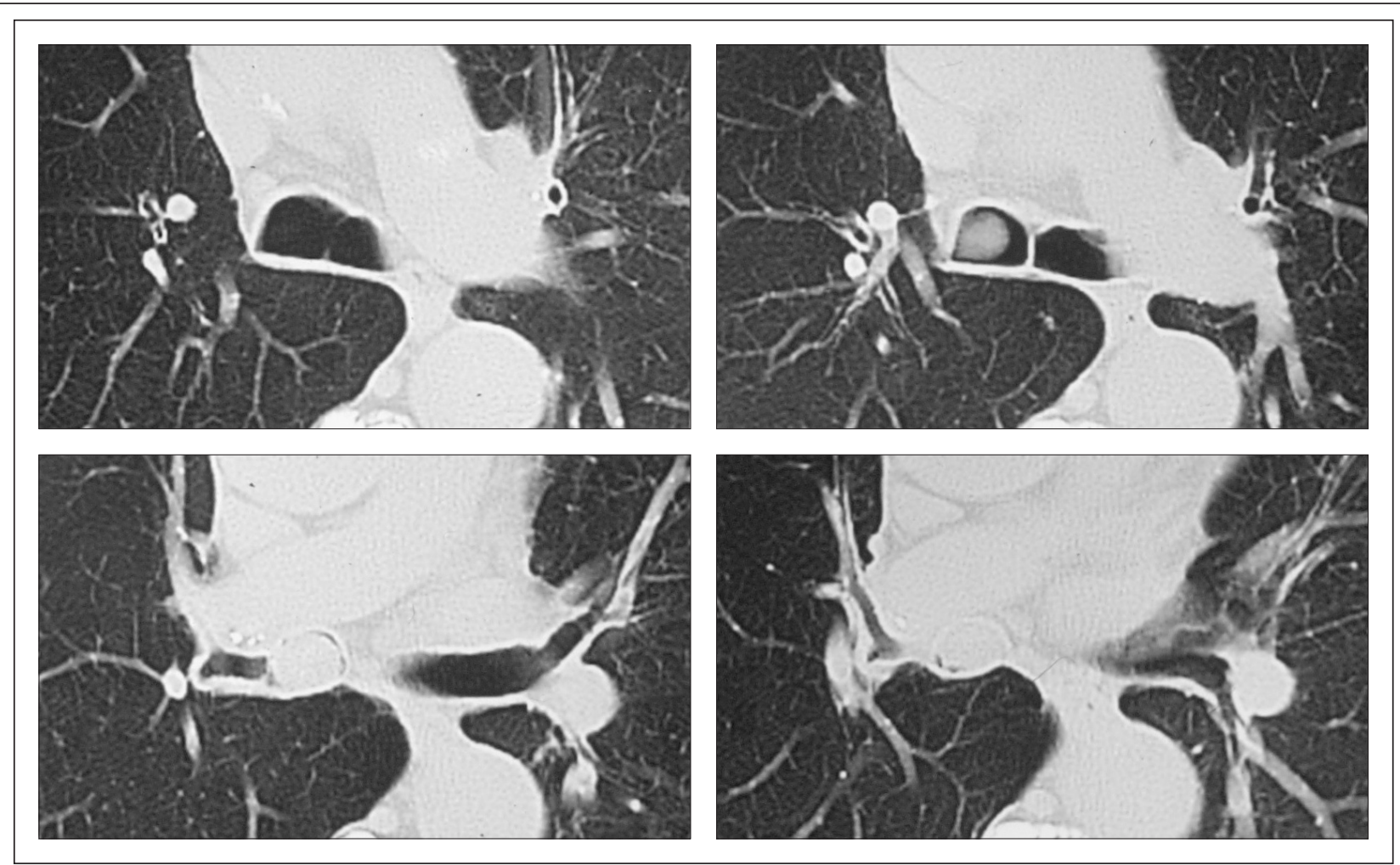

Fig. 1. - A series of contrast-enhanced axial CT scans shows on the right side a well defined endobronchial, pedunculated, smooth surface lesion causing high grade stenosis of the main and intermediate bronchi.
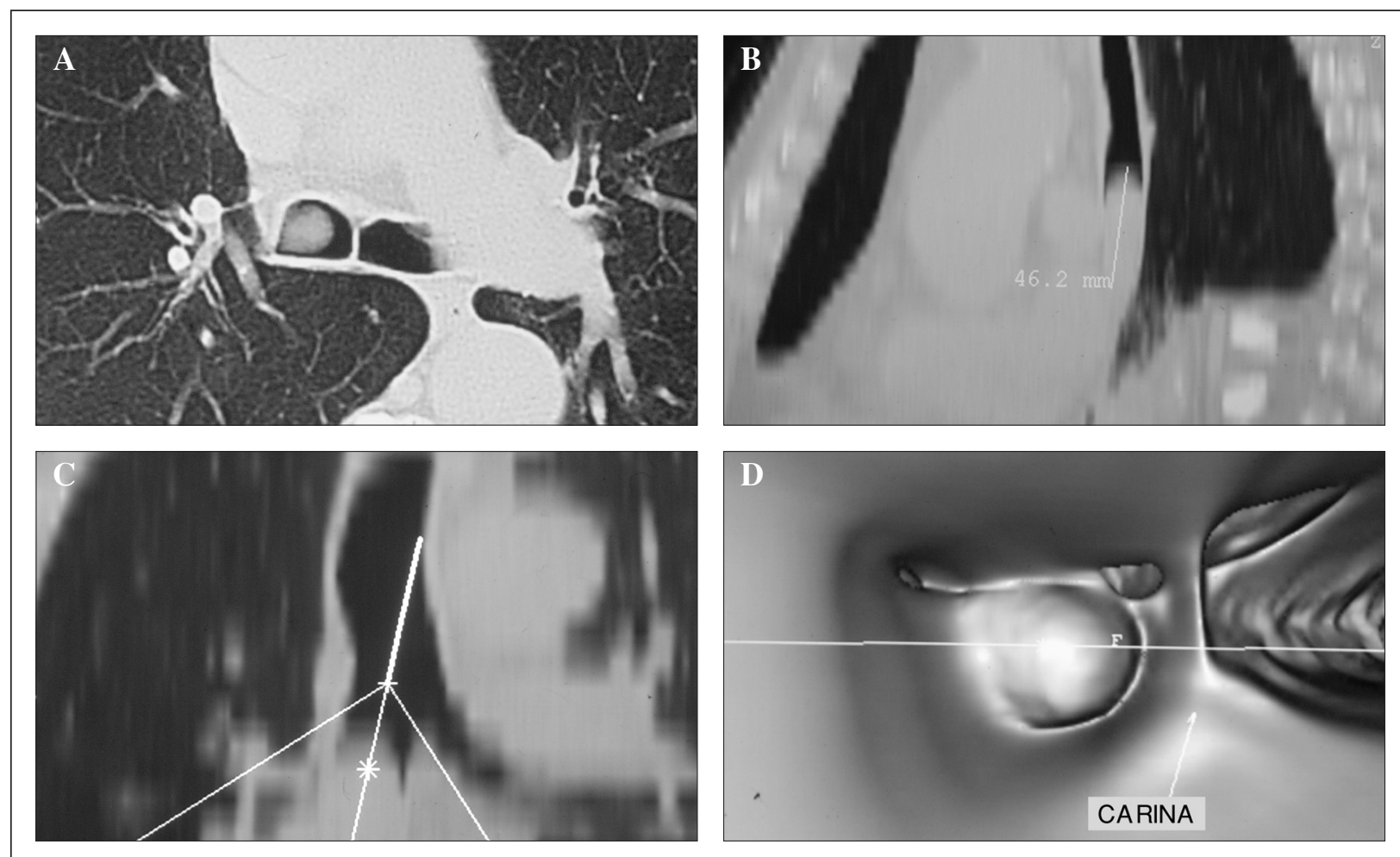

Fig. 2. - Contrast-enhanced axial CT image (panel A) and sagittal MPR (panel B) show the endobronchial lesion extending for $4,6 \mathrm{~cm}$. Coronal image (panel C) demonstrate the pathway followed to obtain virtual bronchoscopic reconstruction (panel D)

tion and Management (DICOM) format on a $\mathrm{O} 2$ Silicon Graphics work station, equipped with a volumetric reconstruction software (Voyager). In addition to axial images, multiplanar reconstruction were obtained and displayed; finally virtual endo- scopic images were obtained with volume reconstruction techniques and threshold value of representation of air-mucosa interface set at $-500 \mathrm{HU}$.

The localisation of the lesion, as well as the relationship with inner bronchial surface and the 


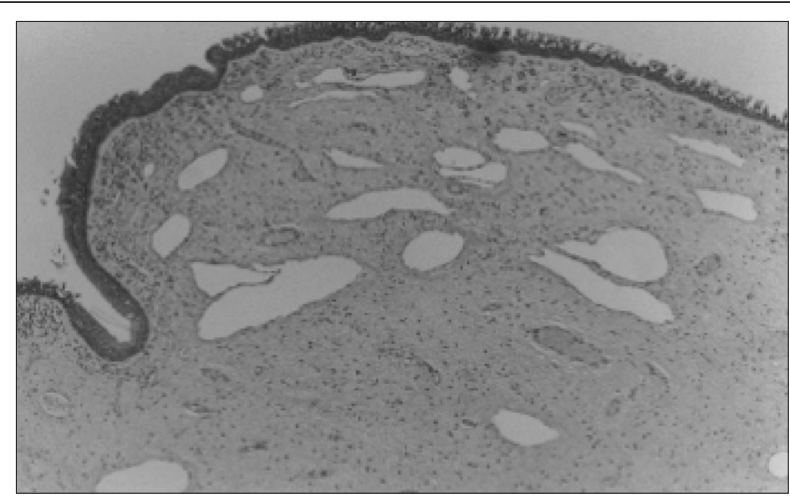

Fig. 3. - Photomicrograph of the specimen (hematoxylin-eosin stain) shows the fibrovascular core of the lesion lined by respiratory epithelium: within this core several capillaries may be observed.

cranio-caudal extension were well documented by the virtual approach, integrated by the axial and multiplanar images (figure 2): the endobronchial lesion extended over $4.6 \mathrm{~cm}$. The virtual bronchoscopy of the bronchial tree distal to the lesion showed the normal appearance of the middle and the lower bronchi.

Since the macroscopic and radiographic characteristics of lesion seemed to be as non-malignant, a treatment with Nd-YAG laser was performed in order to relieve the obstruction. During the first laser treatment the obtained surgical specimen appeared as a compact sample $(\mathrm{cm} 3 \times 0.6 \times$ 0.4 in size) with slightly lobulated surface. The picture of fibrovascular polyp was interpreted as compatible with capillary hemangioma (figure 3). The patient was discharged 10 days after the admission. After 14 days, laser treatment was repeated twice more in order to completely relieve the obstruction.

\section{Discussion}

Lesions in the airways labelled as hemangiomas are relatively common, particularly in childhood, and account for $7 \%$ of all benign neoplasms [5]. True hemangiomas should be differentiated as benignat neoplasms of vascular origin: they are usually made of well formed vascular channels with proliferation of well differentiated endothelial cells [5].

Solitary endobronchial localisation in adults is particularly rare and diagnosis may be difficult, given the lack of specificity of the clinical picture. Wigton and Roatgi reported a case of isolated sessile capillary hemangioma $(1 \times 2 \mathrm{~mm}$ in size $)$ of right upper lobe bronchus, placed one $\mathrm{cm}$ far from the carina [2]. Later Strausz and Soltesz reported two cases: a $2 \times 2 \mathrm{~mm}$ lesion on the ventral wall of middle trachea and a $5 \times 4 \mathrm{~mm}$ one on the carina [3]. Because of the flattened shape and the limited size these lesions did not cause any significant ob- struction. Overall eight cases have been reported in the literature with different morphology [6]: only in one previous case a pedunculated hemangioma obstructing the left lower lobe was reported [7]. The present case is characterised by a late onset presentation with non-specific symptoms masked by the underlying smoke-related chronic airway obstruction. The unusual polipoid appearance added complexity to the problem of differential diagnosis $[2,3,6]$. Surgical excision of such tumors is associated with a favourable prognosis [7]. However, for a better planning of the surgical approach to endobronchial masses completely occluding the airway lumen it is important to evaluate, beside the exact location, the full size and the relationship with adjacent structures. A stepwise diagnostic approach based on spiral CT scan, integrated with tridimensional reconstructions and virtual bronchoscopy, proved particularly suitable for this purpose, confirming the result of previous experiences carried out in different contexts [810]. A larger use of these ancillary imaging techniques must be encouraged, since, in comparison with conventional CT approach, they greatly expand the diagnostic yield at a reasonable additional cost.

\section{References}

1. Kaiser K, Zink S, Herth F, Dienemann H, Schrod L, Gabius HJ. Endobronchial juvenile hemangioma a case of a neonate including immunohistochemical monitoring and nuclear, cellular, and vascular morphometry. Virchow Arch 2001; 438: 192-197.

2. Wigton RB, Rohatgi PK. Isolated bronchial capillary hemangioma: a rare benign cause of hemoptysis. South Med J 1979; 72: 1339-1340.

3. Strausz J, Soltesz I. Bronchial capillary hemangioma in adults. Pathol Oncol Res 1999; 5: 233-234.

4. Paul KP, Borner C, Muller KM, Vogt-Moykopf I. Capillary hemangioma of the right main bronchus treated by sleeve resection in infancy. Am Rev Respir Dis 1991; 143: 876-879.

5. Schoen FJ. Emangioma. In: Cotran RS., Kumar V, Robbins SL eds. Le basi patologiche delle malattie. Padova: Piccin Nuova Libreria, 1997: 577-579.

6. Rose AS, Mathur PN, Endobronchial capillary hemangioma: case report and review of literature. Respiration 2008; 76: 221-224.

7. Harding IR, Williams J, Seal RM. Pedunculated capillary haemangioma of the bronchus. Br J Dis Chest 1978; 72: 336-342.

8. Lesbros D, Ferran JL, Rieu D, et al. Localized hemangioma of the bronchial trunk of the left lung in a 2 month old infant. Arch Fr Pediatr 1976; 33: 895-903.

9. Rogalla P, Meiri N. Virtual endoscopy of the trachea and bronchi. In: Rogalla P, Terwisscha van Scheltinga J, Hamm B eds. Virtual endoscopy and related 3D techniques. Berlin: Springer Verlag 2001: 45-75.

10. Fleiter T, Merkle EM, Aschoff AJ, et al. Comparison of real-time and virtual bronchoscopy in patients with bronchial carcinoma: opportunities and limitations. AJR 1997; 169: 1591-1595. 